\title{
PARTICLE PRODUCTION AND RADIATION ENVIRONMENT AT A NEUTRINO FACTORY TARGET STATION*
}

\begin{abstract}
N. V. Mokhov ${ }^{\dagger}$, FNAL,
Abstract

Efficient production and collection of a large number of muons is needed to make a neutrino factory based on a muon storage ring viable. The results of extensive MARS simulations are reported for Megawatt proton beams on a carbon rod and mercury jet in a 20-T hybrid solenoid, followed by a matching section and decay channel. Beam energy and power in a 2 to $30 \mathrm{GeV}$ range, beam spot size, beam and target tilt angle, target material and dimensions, and capture system parameters are optimized to get maximum muon yields at the end of the decay channel. Other particles transported down the beam line are also studied for the purpose of beam instrumentation. Prompt and residual radiation distributions are calculated and analysis of target integrity, quench stability and dynamic heat load to the superconducting coils, radiation damage and activation of materials near the beam is performed. Absorption of showers in the direction of a primary beam is considered.
\end{abstract}

\section{MARS MODELING}

To achieve adequate parameters of a neutrino factory based on a muon storage ring $[1,2]$ it is necessary to produce and collect large numbers of muons. The system starts with a proton beam impinging on a thick target sitting in a high-field solenoid (20 T, about 1-m long, aperture radius $R_{a}=7.5 \mathrm{~cm}$ ), followed by a matching section and a solenoidal decay channel $(1.25 \mathrm{~T}, 50-100 \mathrm{~m}$ in length, $R_{a}=30 \mathrm{~cm}$ ) which collects muons resulting from pion decay. Optimization of beam, target and solenoid parameters was done over the years with the MARS code [3] for a $\mu^{+} \mu^{-}$collider and a neutrino factory (see bibliography in Ref. [4, 5]).

List of targetry issues includes $\pi / \mu$ production, other particles transported down the beamline, superconducting (SC) coil quench stability, heat loads, radiation damage and activation of materials near the beam, spent proton beam, and numerous shielding issues from prompt radiation to ground-water activation. All these issues were addressed in detailed MARS simulations. Realistic 3-D geometry together with material and magnetic field distributions based on the solenoid magnet design optimization have been implemented into MARS. Graphite (C) and mercury $(\mathrm{Hg})$ tilted targets were studied. A two interaction length target $(80 \mathrm{~cm}$ for C of radius $R_{T}=7.5 \mathrm{~mm}$ and $30 \mathrm{~cm}$ for $\mathrm{Hg}$ of $R_{T}=5 \mathrm{~mm}$ ) is found to be optimal in most cases, keeping $R_{T} \geq 2.5 \sigma_{x, y}$, where $\sigma_{x, y}$ are the beam RMS spot sizes.

\footnotetext{
* Work supported by the Universities Research Association, Inc., under contract DE-AC02-76CH03000 with the U. S. Department of Energy.

†mokhov@fnal.gov
}

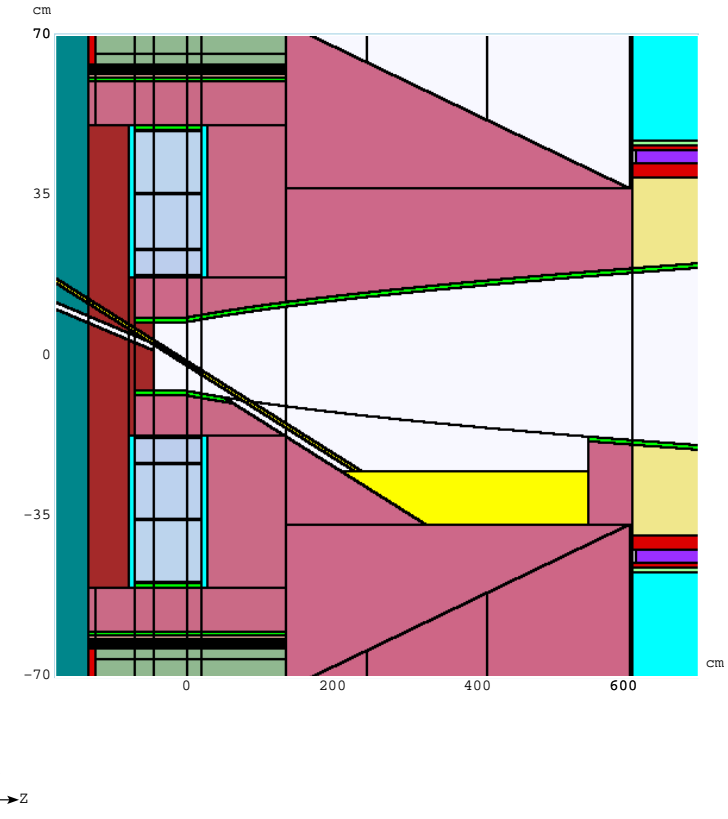

Figure 1: A fragment of the MARS model of target/capture system with tilted proton beam and mercury jet.

The optimized configuration for the Study-2 [2], designed for a $1 \mathrm{MW}$ proton beam of $24 \mathrm{GeV}$ energy (upgradable to $4 \mathrm{MW}$ ), is shown in Fig. 1. The beam intensity is $1.7 \times 10^{13} \mathrm{ppb} \times 6 \times 2.5 \mathrm{~Hz}=2.55 \times 10^{14} \mathrm{p} / \mathrm{s}$, resulting in $5.1 \times 10^{21} \mathrm{p} / \mathrm{yr}$ at $2 \times 10^{7} \mathrm{~s} / \mathrm{yr}$. The model was optimized for $-2<\mathrm{z}<36 \mathrm{~m}, \mathrm{r}<1.8 \mathrm{~m}$. It includes sophisticated coil shielding: water-cooled tungsten-carbide balls at $\mathrm{z}<6 \mathrm{~m}$ and water-cooled copper at $\mathrm{z}>6 \mathrm{~m}$. A proton beam ( $\sigma_{x}=\sigma_{y}=1.5 \mathrm{~mm}, \sigma_{z}=3 \mathrm{~ns}, 67 \mathrm{mrad}$ ) interacts with a $5 \mathrm{~mm}$ radius mercury jet tilted by $100 \mathrm{mrad}$, which is ejected from the nozzle at $\mathrm{z}=-60 \mathrm{~cm}$, crosses the $\mathrm{z}$-axis at $\mathrm{z}=0 \mathrm{~cm}$, and hits a mercury pool at $z=220 \mathrm{~cm}, x=-25 \mathrm{~cm}$. With such a beam-jet crossing, about $97 \%$ of protons have a probability to interact with target material, generating pions and resulting in significant energy deposition in material (Fig. 2) that can at some conditions destroy solid or liquid target. A 8$\mathrm{cm}$ wide mercury pool $(210<\mathrm{z}<550 \mathrm{~cm})$ is a core of a sophisticated spent beam absorber. A 2-mm beryllium window at $\mathrm{z}=610 \mathrm{~cm}$ withstands beam-induced heating (with appropriate cooling), but its lifetime is an issue because the absorbed dose in its center reaches tens of GGy/yr.

\section{PARTICLE PRODUCTION}

Detailed optimizations were performed for the particle yield $Y$, defined as a sum of the numbers of $\pi, K$ and $\mu$ of a given sign and energy interval at the downstream end of the considered system. It turns out that for pro- 


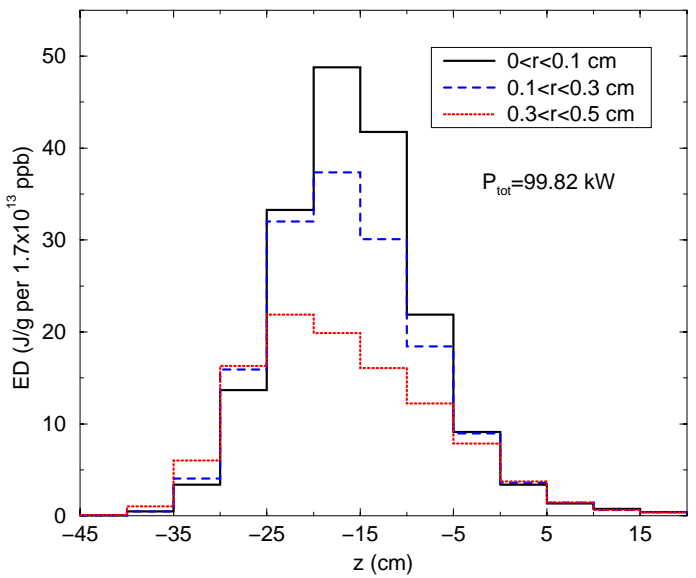

Figure 2: Longitudinal profiles of the energy density deposited in the mercury jet target in three radial regions.

ton energies $E_{p}$ from a few $\mathrm{GeV}$ to about $30 \mathrm{GeV}$, the shape of the low energy spectrum of such a sum is energyindependent and peaks around $\mathrm{E}=130 \mathrm{MeV}$, where $\mathrm{E}$ is $\pi / \mu$ kinetic energy. For the given parameters, the interval of $30 \mathrm{MeV}<\mathrm{E}<230 \mathrm{MeV}$ around the spectrum maximum is considered as the one to be captured by a phase rotation system. The yield $Y$ grows with $E_{p}$, is almost materialindependent at low energies and grows with target $A$ at high energies, being almost a factor of two higher for $\mathrm{Hg}$ than for $\mathrm{C}$ at $E_{p}=16-30 \mathrm{GeV}$ (Fig. 3). It is interesting that the yield per beam power, i.e., $Y / E_{p}$ has a broad maximum around $6 \mathrm{GeV}$. For a 1 to $2 \mathrm{GeV}$ proton beam (CERN, SNS), the optimal target material, from the pion production point of view, is carbon with significantly lower $\pi^{-}$production compared to $\pi^{+}$. To avoid absorption of spiraling pions by target material, the target and beam are tilted by an angle $\alpha$ with respect to the solenoid axis. The yield is higher by up to $30 \%$ for the tilted target with a broad maximum around $\alpha=100 \mathrm{mrad}$. Maximum yield occurs at target radius $R_{T}=7.5 \mathrm{~mm}$ for $\mathrm{C}$ and $R_{T}=5 \mathrm{~mm}$ for $\mathrm{Hg}$ targets with $R_{T}=$ $3.5 \sigma_{x, y}$ and $R_{T}=4 \sigma_{x, y}$ conditions for the beam spot size, respectively. The baseline criterion $R_{T}=2.5 \sigma_{x, y}$ reduces the yield by about $10 \%$ for the graphite target, but is more optimal from the energy deposition point of view. The use of a realistic 3-D magnetic field map in simulations results in the reduction of the $\pi+\mu$-yield in the decay channel by about $7 \%$ for $\mathrm{C}$ and by $10-14 \%$ for $\mathrm{Hg}$ targets, compared with a simple-minded $B_{z}(r, z)$ model.

The optimized results for the yield per a proton on target, for Study-1 ( $16 \mathrm{GeV}$ on C) are $Y_{\pi^{+}+\mu^{+}}=0.18$ and $Y_{\pi^{-}+\mu^{-}}$ $=0.15$ at $\mathrm{z}=9 \mathrm{~m}$, and for Study $-2(24 \mathrm{GeV}$ on $\mathrm{Hg}$, more realistic geometry and field) are $Y_{\pi^{+}+\mu^{+}}=0.40$ and $Y_{\pi^{-}+\mu^{-}}$ $=0.39$ at $\mathrm{z}=36 \mathrm{~m}$. There are substantial fluxes of accompanying particles in the system, which should be taken into account in designing beam instrumentation. In the aperture of the Study-2 channel, at the end of the matching region $(\mathrm{z}=18.6 \mathrm{~m})$, the numbers of particles per proton are $1.03(\mu)$, $1.15\left(p+\pi^{ \pm}\right), 0.07\left(e^{ \pm}\right), 0.02(n)$ and $0.46(\gamma)$.

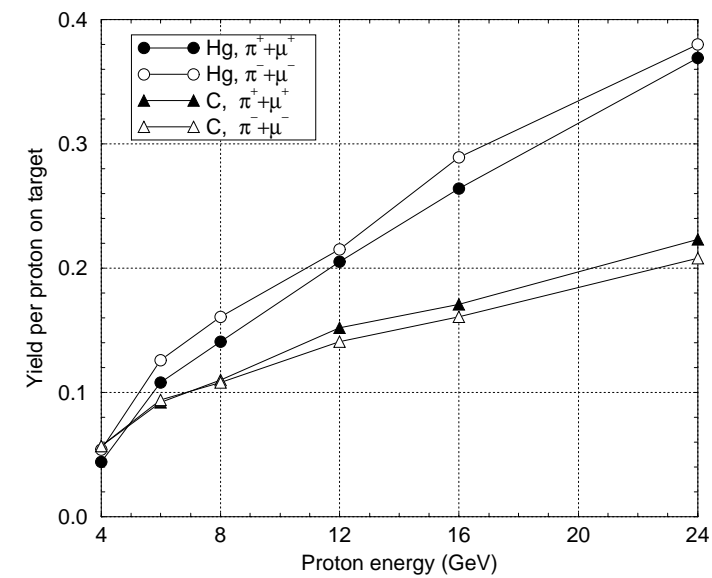

Figure 3: $\pi+\mu$ yield from $\mathrm{Hg}$ and $\mathrm{C}$ targets $v s$ proton energy.

\section{RADIATION FIELDS}

Hadronic and electromagnetic showers are induced in the target and capturing system, resulting in particle fluxes and accumulated dose in system components which can deteriorate their performance rapidly. The SC coils are to be adequately protected to provide their short and long term operation. A carefully designed coil shielding consists of two parts (Fig. 1): 1) at $\mathrm{z}<6 \mathrm{~m}$ it is made of tungstencarbide balls ( $80 \%$ filling factor) cooled by circulating water (WCW), placed in front of the SC coils SC1-SC2 in the 20-T region and SC3-SC6 in the matching section, and surrounds the resistive coils and the spent beam absorber; 2) at $\mathrm{z}>6 \mathrm{~m}$ it is made of copper (70\% filling factor) cooled by circulating water, and protects the potted SC7-SC12 coils in the matching section and further in the straight 1.25-T decay channel (SC13). The calculations show that it does an excellent job in protecting the SC coils against radiation.

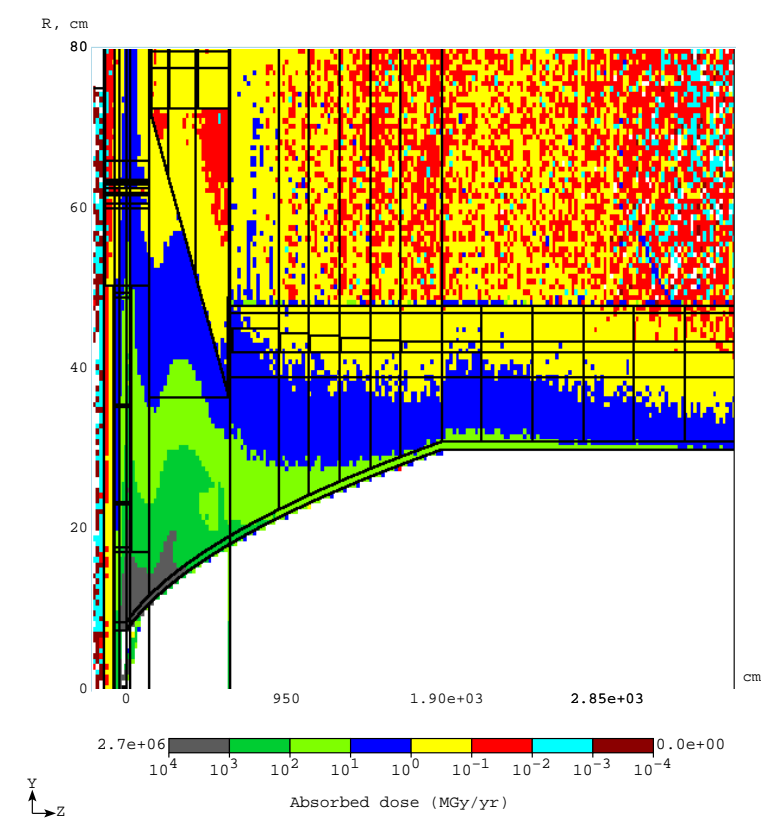

Figure 4: Absorbed radiation dose (MGy/yr) in target/capture system components. 
Table 1: Maximum radiation doses per $2 \times 10^{7} \mathrm{~s} / \mathrm{yr}$ and $1 \mathrm{MW}$ lifetimes of some components of the target system.

\begin{tabular}{|l|c|c|c|}
\hline Component & $\begin{array}{c}\text { Dose/yr } \\
\text { (MGy) }\end{array}$ & $\begin{array}{c}\text { Limit } \\
(\mathrm{MGy})\end{array}$ & $\begin{array}{c}\text { Life } \\
(\mathrm{yr})\end{array}$ \\
\hline Inner shielding & $5 \times 10^{4}$ & $10^{6}$ & 20 \\
Hg containment & $2 \times 10^{3}$ & $10^{5}$ & 50 \\
Hollow conductor & $1 \times 10^{3}$ & $10^{5}$ & 100 \\
Superconducting coil & 6 & $10^{2}$ & 16 \\
\hline
\end{tabular}

The hottest regions in the system are the one at the downstream end of the target at the transition from the 20-T region to a matching section and at a primary beam dump at $\mathrm{z} \approx 4 \mathrm{~m}$ (Figs. 1 and 4 ). The shielding reduces the peak power density to less than $0.3 \mathrm{~mW} / \mathrm{g}$ (below the quench limit) in these two regions as well as in the entire system. The shielding provides also acceptable integrated levels of the absorbed dose (Fig. 4 and Table 1) and particle fluxes (Fig. 5) in the hottest spots, equalizing these to even lower levels in the rest of the system. As Table 1 shows, estimated lifetimes of the critical components are quite satisfactory. The component lifetimes are four times shorter for a $4 \mathrm{MW}$ beam. In the Study-1 design [1, 5], the annual hadron flux in a stationary graphite target is $\sim 5 \times 10^{21} \mathrm{~cm}^{-2}$ which corresponds to several month lifetime. The annual hadron flux $(\mathrm{E}>0.1 \mathrm{MeV})$ and dose in the hottest spot of the inner resistive coil are $1.2 \times 10^{20} \mathrm{~cm}^{-2}$ and $3 \times 10^{10} \mathrm{~Gy}$, respectively.
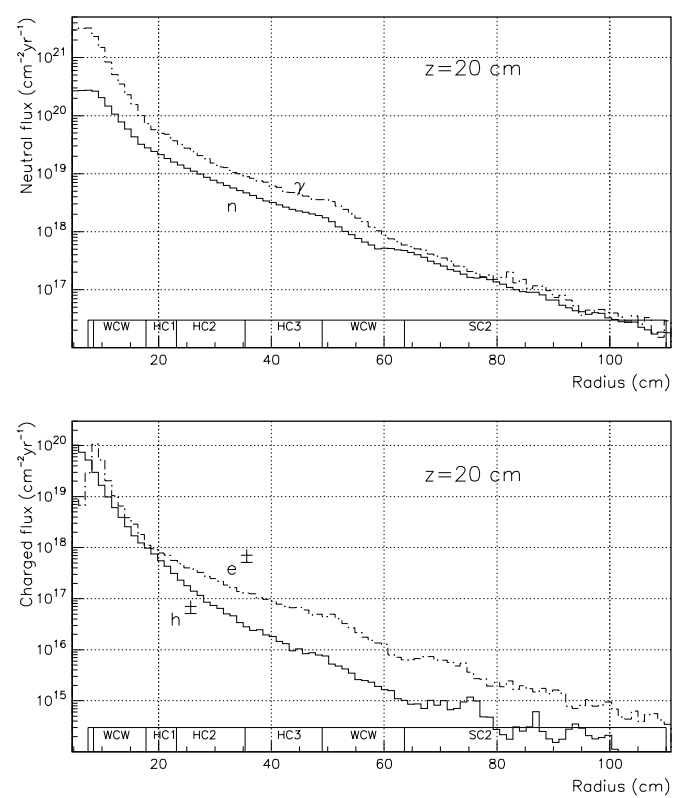

Figure 5: Radial distribution of neutral (top) and charged (bottom) particle fluxes $\left(\mathrm{cm}^{-2} \mathrm{yr}^{-1}\right)$ in 20-T solenoid components at the downstream end of the target.

Heat loads to the main components of the Study-2 design, calculated for a $1 \mathrm{MW}$ beam $(0.979 \mathrm{MW}$ to be exact), are shown in Table 2. About $12 \%$ of the beam power are deposited in mercury (jet plus pool), $50 \%$ in the coil shielding, $1 \%$ in resistive hollow conductor, and only about $0.1 \%$ in the high-field and potted SC coils. About 20\% dissipate in other components and leak from the system. As Fig. 6 shows, the inner shielding becomes extremely radioactive, with residual dose rate up to $1 \mathrm{kSv} / \mathrm{hr}$. This will require remote control and robotics for the inner parts of the system. It drops by two orders of magnitude after several weeks. The residual dose outside the cryostat is significantly lower, of the order of $100 \mathrm{mSv} / \mathrm{hr}$. Radiation shielding needed is about $2 \mathrm{~m}$ of steel followed by concrete blocks to protect ground water followed by several meters of concrete and dirt to provide personnel protection.

Table 2: Power dissipation in the main target/capture system components.

\begin{tabular}{|l|c|}
\hline Component & Total heat load $(\mathrm{kW})$ \\
\hline Mercury & 119.181 \\
1-cm inner vessel & 113.873 \\
WCW shielding & 489.118 \\
Cu-water shielding & 12.939 \\
Hollow conductor & 9.910 \\
SC1-SC2 & 1.256 \\
SC3-SC13 & 1.385 \\
\hline
\end{tabular}

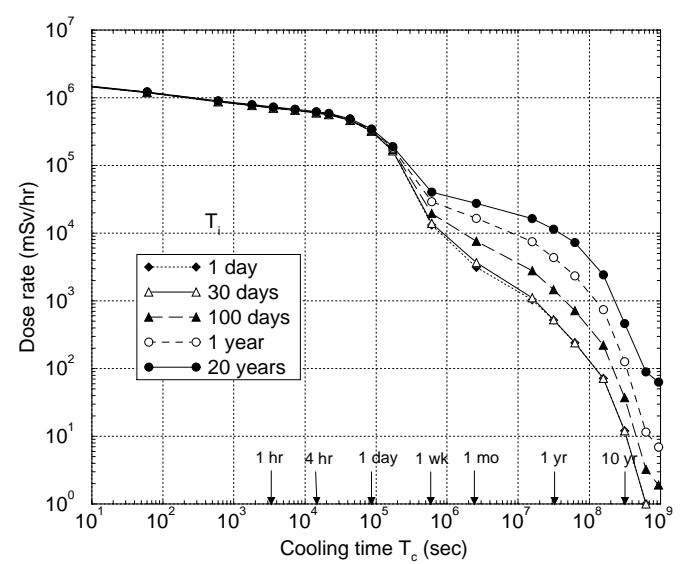

Figure 6: Residual dose rate $(\mathrm{mSv} / \mathrm{hr})$ in the innermost tungsten-carbide shielding around the target $v s$ cooling time for several irradiation times.

\section{REFERENCES}

[1] N. Holtkamp and D. Finley, eds., A Feasibility Study of a Neutrino Source Based on a Muon Storage Ring, Fermilab-Pub00/108-E, 2000.

[2] http://www.cap.bnl.gov/mumu/studyii/FS2-report.html.

[3] N. V. Mokhov, "The MARS Code System User's Guide", Fermilab-FN-628, 1995; N. V. Mokhov and O. E. Krivosheev, "MARS Code Status", Fermilab-Conf-00/181, 2000. http://www-ap.fnal.gov/MARS/.

[4] N.V. Mokhov, "Particle Production for a Muon Storage Ring: I. Targetry and $\pi / \mu$ Yield", Neutrino Factory-2000, Monterey, CA, May 2000; Fermilab-Conf-00/208, 2000.

[5] N.V. Mokhov, "Particle Production for a Muon Storage Ring: II. Radiation Loads", ibid, Fermilab-Conf-00/209, 2000. 